\title{
Compact Probe for Non-Contact Ultrasonic Inspection with the Gas-Coupled Laser Acoustic Detection (GCLAD) Technique
}

\author{
D. Vangi ${ }^{1} \cdot$ M. Bruzzi ${ }^{2} \cdot$ J. N. Caron $^{3} \cdot$ M. S. Gulino ${ }^{1}$ (I)
}

Received: 10 June 2021 / Accepted: 25 September 2021 / Published online: 21 October 2021

(c) The Author(s) 2021

\begin{abstract}
Background Gas-Coupled Laser Acoustic Detection (GCLAD) is a non-contact ultrasonic detection technique whose functioning relies on the deviation that a probe laser beam sustains when intersected by an acoustic wavefront propagating in a fluid. The maximum sensitivity of the technique is typically obtained when the ultrasound insists on an ample portion of the probe laser beam extension, but such a condition can be unfeasible in several non-destructive testing applications (as in case of limited accessibility to the component).

Objective In the present work, a solution is provided enabling transformation of the GCLAD device in a point detector. This is based on the use of two mirrors for confining the laser beam in an area with limited width and depth, where reflections however maximize the portion of the probe laser beam subjected to ultrasonic oscillation.

Methods The characteristics of the obtained GCLAD probe are thoroughly analysed by applying the device to the detection of surface acoustic waves, propagating on a metal bar and refracting into the air. Two different inspection configurations are considered, whose difference lies in the mutual orientation between laser beam and solid surface. The effect on the received signal amplitude of the number of beam reflections, the dimensions of the resulting device, and the bar axisimmetry is investigated in both configurations.

Results The optimization of all the analysed standpoints enables obtaining a compact GCLAD probe that features the same signal amplitude of the non-compact alternative. To obtain maximum responsivity of the system, the number of reflections must be maximized, while the distance between the mirrors must be carefully set based on the employed inspection configuration and the eventual axisimmetry of the specimen. The devised GCLAD compact probe is capable of expanding the application range of the technique also to those cases in which the use of point detectors is desirable, without compromising the signal-to-noise ratio of the resulting acquisitions compared to the non-compact alternative.
\end{abstract}

Keywords Surface acoustic wave (SAW) - Bidimensional components · High reflection mirror · Cylindrical wavefront . Point detection

\section{Introduction}

The Gas-Coupled Laser Acoustic Detection (GCLAD) technique belongs to the class of optical, non-contact methods for ultrasonic wave detection [1]. The GCLAD technique

M. S. Gulino

michelangelosanto.gulino@unifi.it

1 Department of Industrial Engineering of Florence, Universita degli Studi di Firenze, Via di Santa Marta 3, Firenze 50139, Italy

2 Department of Physics and Astronomy, Universita degli Studi di Firenze, Via Sansone 1, Sesto Fiorentino 50019, Italy

3 Research Support Instruments, 4325-B Forbes Boulevard, Maryland 20706, Lanham, USA is based on the deviation of an optical beam when the laser light passes through fluctuations in the refractive index caused by ultrasonic waves, moving typically in the air from a solid surface; the total deviation of the probe laser beam is detected by a position-sensitive photodetector [2, $3]$. Such technique is extremely simple and economical from the required instrumentation standpoint, exhibiting also the advantage of being almost uninfluenced by the component's surface finishing or reflectivity [4] in contrast to other optical detection techniques [5, 6]; it has been shown that the GCLAD is characterized by a higher sensitivity to that of a Fabry-Pérot confocal interferometer for ultrasonic signals in the range between $2 \mathrm{MHz}$ and $7 \mathrm{MHz}$, even if one order of magnitude lower for a frequency of $500 \mathrm{kHz}$ [7]. The technique features a wide frequency band that enables high 
resolution in time of the detected echoes, as well as detecting higher energetic components for broadband ultrasonic sources (as in the case of excitation by photoacoustic signals from pulsed or modulated lasers [8-15]).

To date, no significant application of the GCLAD exists in the non-destructive testing (NDT) field; nevertheless, the characteristics of the technology and the suitability in detecting defects have been demonstrated by [16]: signals received by Air-Coupled Transducers (ACTs) are less noisy, but the amplitude for the GCLAD is up to 100 times higher than that for an ACT. The technique can be exploited in various configurations, depending on the geometry of the component and the type of wave (surface or bulk) refracted in the fluid (air or liquid) surrounding the piece [16]. Some arrangements of the laser beam with respect to the component allow a line inspection, i.e., detecting any defect present along a complete line of the piece without the need to scan the laser source along such line [17]. This peculiarity makes the GCLAD potentially advantageous compared to techniques where an $x-y$ scan of the entire surface is required, as only scanning along one direction of the piece is necessary. In addition, the GCLAD can enable effectively using a signal amplification effect deriving from constructive interference (occurring in the air) between surface waves propagating in air and that reflected by a surface defect. This effect, clearly visible a few millimetres from the surface of the piece, allows the component to be inspected with techniques like scanning laser source (SLS) or scanning laser line source (SLLS) [9, 18, 19], without the need to observe echoes caused by the defect but only the primary wavefront, with an evident increase in reliability in the detection of defects.

The curvature and displacement of the laser beam depend on the refractive index gradient, generated by the pressure variations within the wave refracted in the fluid. The total deviation of the laser beam in correspondence of the photodetector results from the contribution of all the displacements and deflections sustained by the beam during its path within the area where the refractive index is altered. This total deviation, therefore, also depends on the length of the optical beam path within the area characterized by the pressure gradient. Generally, therefore, the greater the length of the area affected by the pressure wave intersected by the laser beam the greater the response of the system. Such a feature of the system can also be advantageous for applications such as line inspections, where typically vast areas exist in which the refracted wave propagates in the fluid. In other applications this feature can be a disadvantage, e.g., if point detection on the component of a pressure wave or defect echo is sought, the detection must be restricted to a narrow region. In such a case, the laser beam traverses only a limited area in which the refractive index is altered and the GCLAD response is proportional to such an extension
[17]. Another example is represented by conditions in which curved components should be interrogated. In this case, the pressure wave refracted in the fluid has non-flat wavefronts and the laser beam crosses multiple wavefronts, encountering pressure gradients with opposite sign. The curvature and displacement of the beam hence tend to compensate and, at the exit of the beam from the altered area, the total deviation is typically low although not zero [20]. Another eventuality is represented by limited space to access the component which complicates the monitoring, such as on rotating shafts on which elements are located in mutually close points (e.g., brake discs and wheels mounted on a railway axle [21]).

The present work aims at overcoming these limitations of the GCLAD technique, to obtain a high response of the system even when sensing is performed on small regions of the component. In particular, the operating principle of the method and the possible experimental configurations are first briefly described that allow, on small observation areas, the response to be increased. An optimal configuration is then proposed, which enables obtaining high responses with a compact device. This device, once industrialized, would make it possible to employ a "probe" to be handled like a classic piezoelectric, air-coupled transducer.

\section{The GCLAD Technique}

The Gas-Coupled Laser Acoustic Detection (GCLAD) is based on the overall displacement that a laser beam sustains when passing through a pressure field inside a fluid, typically generated by an ultrasonic wave refracted from a solid into the air $[2,20,22]$. The pressure field results in a variable refractive index of the air, consequently displacing the beam as different pressure gradients are intercepted [23]. Considering the sinusoidal pressure field generated by the ultrasonic wave which propagates along the $z$ direction (Fig. 1), the corresponding variable pressure value $p(z, t)$ can be expressed as follows [24]:

$p(z, t)=K \rho_{0} c^{2} \delta \sin (k z-\omega t)$

In Equation (1), $\omega$ is the angular frequency of the ultrasound in the fluid, $\rho_{0}$ the density of the fluid, $c$ the speed of sound in the fluid, $K$ is the wave number so that $K=\omega / c$ and $\delta$ is the surface displacement (which generates the ultrasound in the fluid). Referring once again to Fig. 1, the laser beam propagating along $x$ accumulates deviation along $z$ from its ideal optical path, as it travels through the fluid $x_{s}$-long distance subjected to pressure variation. Indicating as $n_{0}$ the refractive index of the unperturbed fluid and based on the Eikonal equation [25], such $z$ displacement follows the trend of Equation (2) [17]: 


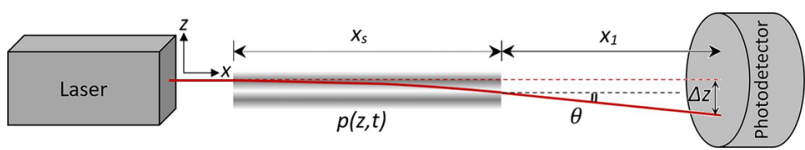

Fig. 1 In the GCLAD, a laser beam is meant to travel in a fluid in which a variable pressure field (ultrasound) is present; the beam sustains deviation from its ideal optical path as soon as it intersects the ultrasound in an $x_{s}$-long path, moving unperturbedly (but with specified angle $\theta$ ) along the $x_{1}$ length. A photodetector ultimately acquires the total $\Delta z$ deviation, transducing it in a proportional voltage difference

$z\left(x_{s}\right)=\frac{\left(n_{0}-1\right)}{2 n_{0} \rho_{0} c^{2}} x_{s}^{2} \frac{\partial}{\partial z} p\left(z_{0}, t\right)$

From Equation (1), the maximum displacement $\Delta z$ in correspondence of the photodiode of a single optical beam is derived:

$\Delta z=\frac{\pi^{2} \delta\left(n_{0}-1\right)}{l^{2} n_{0}} x_{s}^{2}+\theta x_{1}$

where $l$ represents the acoustic wavelength in the fluid, while $\theta=\frac{d}{d x_{s}}\left(z\left(x_{s}\right)\right)$. The deviation of the laser beam is detected by a position-sensitive photodetector and converted into a displacement-proportional voltage difference [22] as follows:

$\Delta V=\frac{-8 G S \kappa P}{\sqrt{\pi^{3}}} \operatorname{erf}\left(\frac{d}{w}\right) \frac{\Delta z}{w}\left[e^{\left(-\frac{d^{2}}{4 w^{2}}\right)}-e^{\left(-\frac{t^{2}}{4 w^{2}}\right)}\right]$

In Equation (3), $G$ is the amplifier gain, $S$ is the resistance employed to convert the current from the photodiode to voltage difference, $P$ the average power of the laser beam at the photodetector, $d$ is the diameter of the photodiode active area, $w$ the beam spot, and $\kappa$ is the photodetector sensitivity given by:

$\kappa=\frac{q \eta}{h v}$

where $q$ is the electronic charge, $\eta$ is the quantum efficiency, $h$ is Planck's constant, and $v$ is the frequency of light.

Along its path in the air, the laser beam diverges and its diameter as a function of the travelled distance can be calculated as follows:

$w=w_{0} \sqrt{1+\left(\frac{\lambda x}{\pi w_{0} n_{0}}\right)^{2}}$

In Equation (6), $w_{0}$ is the original beam diameter and $\lambda$ is the wavelength of the light radiation.

Figure 2 highlights the trend of $\Delta V$ as a function of the $x_{1}$ and $x_{s}$ distances (refer to Fig. 1), obtained by Equation (4) with $\Delta z$ values given by Equation (3) and the values of the beam diameter provided by Equation (6). Calculation considers $G=100, S=100 \mathrm{ohms}, \kappa=0,119 \mathrm{~A} / \mathrm{W}, P=133 \mathrm{~mW}, d=$ $6 \mathrm{~mm}, t=0,25 \mathrm{~mm}, w_{0}=0,5 \mathrm{~mm}, \lambda=6,8 \mathrm{~mm}, \delta=1 \mathrm{~nm}, v=$ $469 \mathrm{THz}$. The solid line curve indicates the signal obtained with $x_{1}=0$, i.e., with the photodetector placed immediately at the end of the $x_{s}$-long area of variable pressure where the acoustic wave propagates. Beyond the area where the pressure is variable, the signal to the photodetector varies solely due to the angle of deflection $\theta$. The other curves are obtained by setting $x_{s}=0.1 \mathrm{~m}, 0.4 \mathrm{~m}$ and $0.8 \mathrm{~m}$. To augment the system sensitivity (i.e., to increase the response
Fig. 2 Amplitude trend of the signal obtained by the photodetector as a function of the $x_{1}$ distance for different values of $x_{s}$

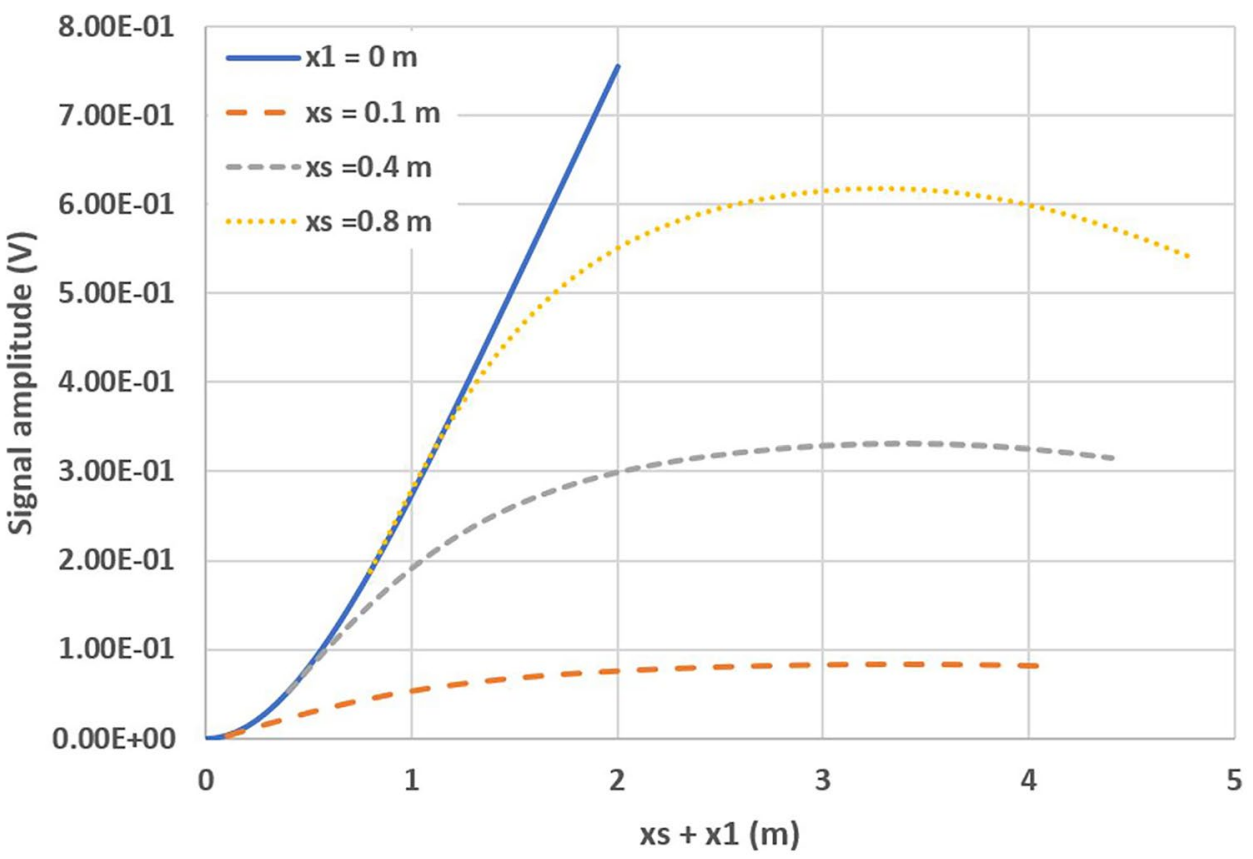




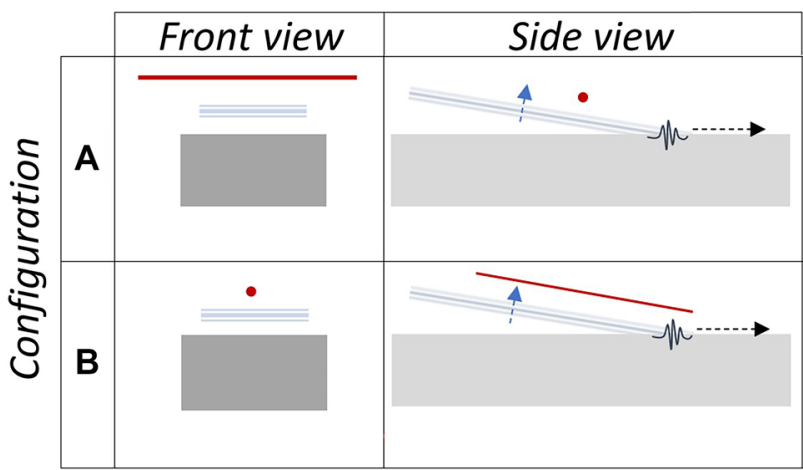

Fig. 3 Experimental configurations for the detection of surface acoustic waves on the component, refracted in the air

of the photodetector), it is possible to increase both $x_{s}$ and $x_{1}$. With higher values of $x_{s}$, increasing deflection angles are obtained and the influence of $x_{1}$ on the response of the system is greater.

To obtain high responses, a significant increase in the distance $x_{s}+x_{1}$ is required; therefore, the distance between the laser and the photodetector should also increase, with disadvantages in terms of size and ease of use. Beyond a certain distance, the signal tends to decrease after reaching a maximum, because the diameter of the optical beam becomes too large compared to the diameter of the photodetector [17].

From Fig. 2, it is derived that it is convenient to increase $x_{s}$ or even to have purely $x_{s}$ and $x_{1}=0$, the distance $x_{s}+$ $x_{1}$ being the same. However, while $x_{1}$ can be varied at will by moving the photodetector away from the point where the pressure wave occurs, the $x_{s}$ measurement depends on the extension of the altered pressure zone or the extension of the acoustic wavefront. The latter is generally small and depends on the size and geometry of the structre in which the ultrasound propagates, the arrangement of the laser beam with respect to the piece and the type/mode of propagation of the ultrasonic wave in the solid. In Fig. 3 some experimental configurations for the detection of the surface ultrasonic waves in the piece are evidenced (similar considerations apply to other types of waves, like bulk waves [16]). Depending on the configuration used and the geometry of the component, different $x_{s}$ lengths can be employed.

Configuration B generally allows obtaining a wavefront in the air which can extend even for the entire length of the structure. The surface wave propagates in the piece and is simultaneously refracted in the air as a longitudinal wave. When the surface wave reaches the end of the piece, the refracted wavefront intercepts the laser beam along the entire length of the solid. The optical beam is oriented to be parallel to the wavefront, a solution that maximizes the system response because the pressure gradient is the same over the entire length of the optical beam. Therefore, if the piece geometry permits it, a high $x_{s}$ length can be obtained in this configuration. The spatial resolution is however limited, that is, retrieval of the ultrasonic wave in the solid does not resemble a point detection and does not allow identification of the wave passage in a specific and restricted area.

It must equally be considered that in this configuration, as the length $x_{s}$ increases, the distance of the laser beam from the surface of the piece also increases, since the beam is inclined by the angle of refraction in the air of the acoustic waves. Therefore, because of the attenuation of the acoustic wave in the air, the amplitude of the signal does not raise as expected based solely on the characteristics of the laser beam, but also because the pressure gradient decreases the further it is moved away from the piece. Conversely, configuration A detects the passage of the ultrasonic wave at the point where the laser beam is positioned; in this case, the $x_{s}$ length is typically limited to the width of the wavefront transversely to its propagation direction.

\section{Compact Probe}

The proposed experimental configuration allows obtaining a high $x_{s}$ and simultaneously achieving a reasonable spatial resolution, comparable with traditional air-coupled probes. The physical principle is the reflection of the laser beam on a pair of parallel and staggered flat mirrors, to achieve a greater optical path within the variable pressure field in a limited space. Figure 4 shows the scheme of the device.

In this way, the length $x_{s}$ is equal to the total path travelled by the optical beam in its reflections between the two mirrors:

$x_{s}=\frac{d(n+1)}{\cos (\beta)}$

where $n$ is the number of reflections, $\beta$ is the beam angle of incidence on the mirrors and $d$ is the distance between the two mirrors. Detection of the ultrasonic signal is obtained on the length defined by the two mirrors, with length $d$ and width $h$ :

$h=d(n+1) \tan (\beta)+w$

The additional term $w$ in Equation (8) accounts also for the finite size and encumbrance of the beam diameter. The angle $\beta$ should be chosen so as $2 d \tan (\beta)>w$, to limit the mutual influence between subsequent reflections. To obtain a limited detection area, both $d$ and $h$ should be low; to achieve an elevated $x_{s}$ value, $n$ should be high and $\beta$ as low as possible, i.e., $\tan (\beta)=\frac{w}{2 d}$.

However, as the optical path and the number of reflections increase, various phenomena occur that reduce the response of the photodetector: 


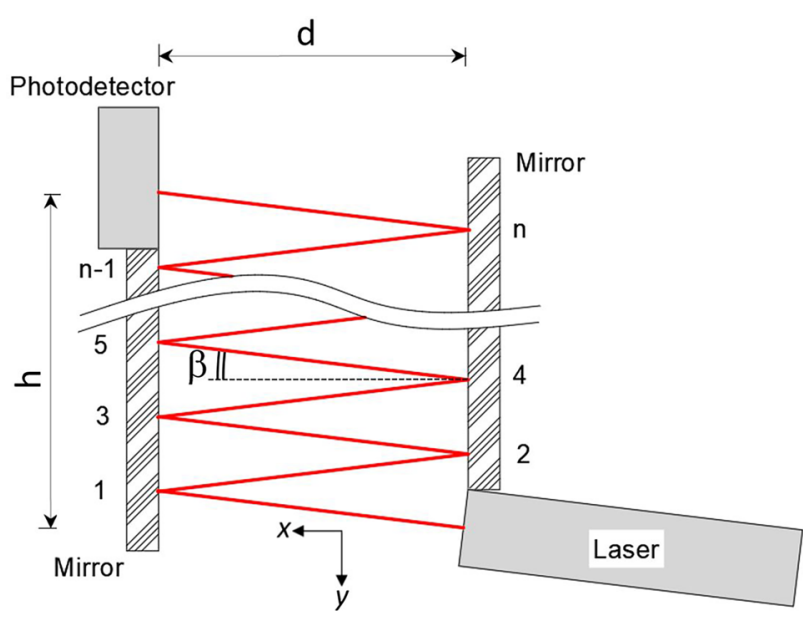

Fig. 4 Scheme of the developed device

a) A loss of power of the optical beam occurs, caused by the reflection coefficient of the mirror different from the unit. This means that after $n$ reflections the power reaching the photodetector is reduced, consequently linearly decreasing the response. This fact can be easily remedied by using a higher initial laser power, which takes into account the losses suffered from each reflection. Given the reflection coefficient for non-polarized waves, in the Schlick approximate form:

$$
R=\left(\frac{n_{1}-n_{2}}{n_{1}+n_{2}}\right)^{2}+\left[1-\left(\frac{n_{1}-n_{2}}{n_{1}+n_{2}}\right)^{2}\right](1-\cos (\beta))^{5}
$$

with $n_{1}$ and $n_{2}$ refractive indices in the air and the mirror; a power $P=P_{0} R^{n}$ will be hence reached in correspondence of the photodetector. Since the refractive indices depend on the wavelength of the employed laser beam, also $R$ will be affected by this wavelength. The limit of the power of the laser beam is provided by the saturation of the photodetector.

b) There is a loss of power of the optical beam arriving at the photodetector caused by the absorption of light in the fluid crossed by the beam. The intensity of the optical radiation is reduced exponentially as a function of the optical path $x$, according to the absorption coefficient $\alpha$ :

$$
I=I_{0} e^{-\alpha x}
$$

c) Diffraction phenomena occur on the mirror surface.

d) Because of the finite beam dimensions, a part of the reflected beam interferes with a part of the incident beam, producing a variable intensity field.

e) As the optical path increases, the beam spot widens because of the divergence and the photodetector response varies following Equation (4).
Therefore, the choice of the number of reflections and the device geometry must be pursued considering all these factors. Moreover, the displacement $\Delta z$ differs from the trend predicted by Equation (3), valid for a displacement of an optical ray of infinitesimal dimensions. In reality, the laser beam has a finite spot size and can be imagined as a set of optical rays distributed on the beam section, each of which intersects an area with a different pressure gradient [17]. The pressure waves in the air, for the most typical frequencies employed in ultrasonic NDT of components, feature wavelengths of less than a millimetre, i.e., comparable with the usual laser beam diameters. Hence, some optical rays encounter pressure gradients with inverse sign compared to others within the beam; the global displacement $\Delta z$ will be averaged over all displacements in each zone of the beam section and the original shape and Gaussian distribution will also change. The phenomenon is influenced by the ratio between the wavelength of the pressure wave and the beam diameter [26], which varies gradually along the optical path. The overall response of the photodetector will therefore be averaged based on the actual displacement and distortion of the beam section.

In light of the many factors that affect the response of the photodetector, it is advisable to perform experimental tests to optimize the number of reflections, the laser power and the distance between the mirrors, depending on the geometries to be obtained in terms of the apparatus dimensions and inspection area.

Finally, to increase the response of the system, it is possible to use an arrangement as shown in Fig. 5, with the photodetector inclined at an angle $\theta$ with respect to the laser beam. In this way, a displacement $\Delta z$ of the laser beam corresponds to a displacement of $\Delta z / \cos (\theta)$ on the photodetector. Experimental tests have been performed considering the absence of mirrors (direct path between the laser source and the photodetector) for $\theta$ values up to $45^{\circ}$; an increase of $40 \%$ has been consequently observed, as analytically predicted. Once implemented in the layout together with the mirrors' supports, the photodetector can however be rotated only if appropriately distanced from the supports, because of its case encumbering (Fig. 6). This entails an overall decrease in the signal amplitude and modifications to the conditions among the diverse tests, which could affect influence assessments for the different parameters under investigation. For this reason and for the sake of simplicity, a $\theta$ value of $0^{\circ}$ has been chosen and employed in the subsequent analyses; nevertheless, once the several parameters has been set after the layout optimization, such a solution provides an efficient way to increase the signal amplitude outputted by the GCLAD.

As regards the sensitivity of the system for the various geometries of the device (length $d$, width $h$ and number of reflections), let us assume a planar acoustic wavefront. In this case, if the laser probe beam lies in a plane parallel 


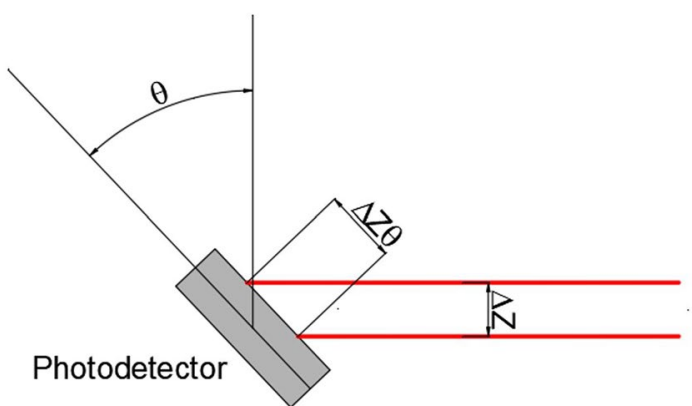

Laser

Fig. 5 Photodetector inclined with respect to the laser beam, to increase the beam deviation detected by the sensor

to the wavefront, the displacement $\Delta z$ of the optical beam depends on the displacement of the surface $\delta$ and on the $x_{s}$ and $x_{1}$ lengths, according to Equation (3). Fixing the surface displacement $\delta$ and considering a negligible and fixed distance $x_{1}$ for the employed device, the displacement of the optical beam depends only on the length of the optical path $x_{s}$ within the acoustic pressure field achieved with the multipass system. Using the proposed device, the optical path is both confined in the space among the mirrors and parallel to the acoustic wavefront (which is supposed to cross the entire device); hence, the response of the system is independent of the optical beam propagation direction and therefore by the configuration A or B employed. For more complex geometries where the wavefront - and consequently the pressure gradient - is not planar, the best solution corresponds to conditions for which the laser beam path intersects regions with a pressure gradient as constant as possible (see Section 5).

The voltage difference $\Delta V$ at the photodetector results from Equation (4), represented by a linear function of $x_{s}$ considering Equation (6). The Signal-to-Noise Ratio (SNR) for a photoconductive photocell can be expressed as reported by [7]:

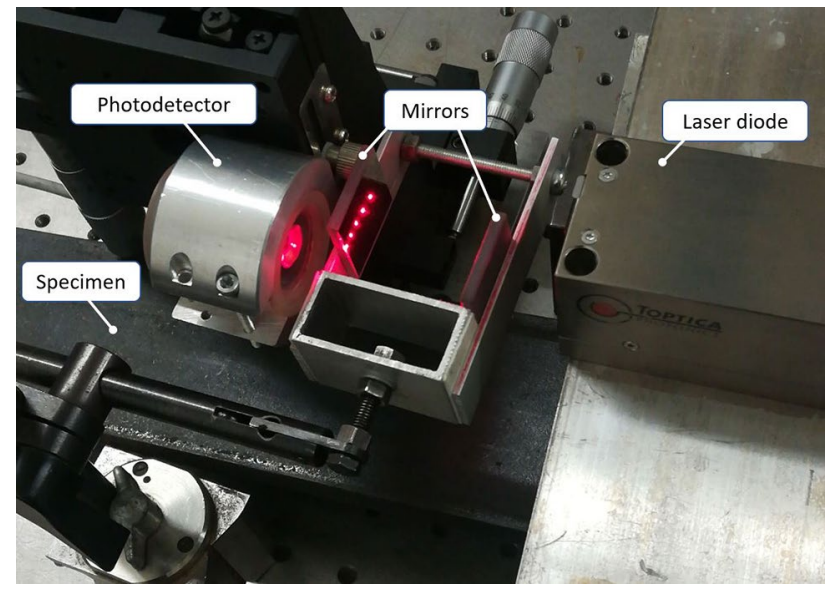

Fig. 6 Experimental setup for the tests on a flat bar
$S N R=\frac{2 P \eta \lambda}{3 \pi h c b}\left(\frac{\Delta z}{w}\right)^{2}$

In Equation (11), $P$ represents the optical signal power and $b$ the ultrasonic frequency band. Considering Equations (3) and (6), this results in:

$S N R=\frac{2 P \eta \lambda}{3 h c b} \frac{\pi^{4} \delta^{2}\left(n_{0}-1\right)^{2} x_{s}^{4}}{n_{0} l^{2}\left(\pi w_{0} n_{0}+\lambda x_{s}\right)}$

The former expression, having referred to Equation (3) that is valid for a single optical beam, is related to the case where the laser beam diameter is negligible compared to the acoustic wavelength $l$. In fact, if the laser beam intersecting the acoustic field has finite dimensions, at each point in the beam section there are different pressure gradients and different displacements as a consequence: the global displacement of the beam is averaged, and the initial circular shape of the beam gets distorted. In this case, in [17] the variation of the average displacement $\Delta z$ at the photodetector is highlighted as a function of the ratio $w_{0} / l$ between the spot diameter and the-mono frequency acoustic wavelength.

\section{Signal Enhancement}

To test the proposed device, preliminary investigations were performed using:

- Two Thorlabs ME1S-PO1 mirrors with a reflection coefficient of 0.955 for a wavelength of $640 \mathrm{~nm}$, with dimensions of 25.4 × $25.4 \mathrm{~mm}^{2}$;

- A TOPTICA iBeam Smart 640 laser diode with a wavelength of $640 \mathrm{~nm}$ and adjustable power from $2 \mathrm{~mW}$ to $100 \mathrm{~mW}$, with an elliptical initial spot measuring $0.7 \mathrm{x}$ $0.2 \mathrm{~mm}^{2}$ ( $x$ and $y$ dimensions).

- A Luna Optoelectronics SD 197-23-21-014 photodetector, a Brüel and Kjær 2638 amplifier and filter.

- A Panametrics piezoelectric probe with a central frequency of $500 \mathrm{kHz}$ (600 kHz bandwidth) mounted on a wedge for the excitation of surface acoustic waves (SAWs [27]) on a specimen;

- A steel bar with a rectangular section $\left(500 \times 80 \mathrm{~mm}^{2}\right)$, employed as specimen for the propagation of SAWs.

Figure 6 shows the experimental setup. The photodetector has been positioned near a mirror, to minimize the distance $x_{1}$ (about $1 \mathrm{~cm}$ ). Based on the angle of the laser beam, several reflections have been obtained between the mirrors before reaching the photodetector. The $x-y$ plane on which the reflections occur has been kept parallel to the acoustic wavefront in the air, inclined at an angle 
Fig. 7 Trend of the $d x$ and $d y$ diameters of the spot as the distance from the laser source is varied; the lines indicate the measurement carried out without mirrors, while the points represent the measurements obtained with reflections on the mirrors

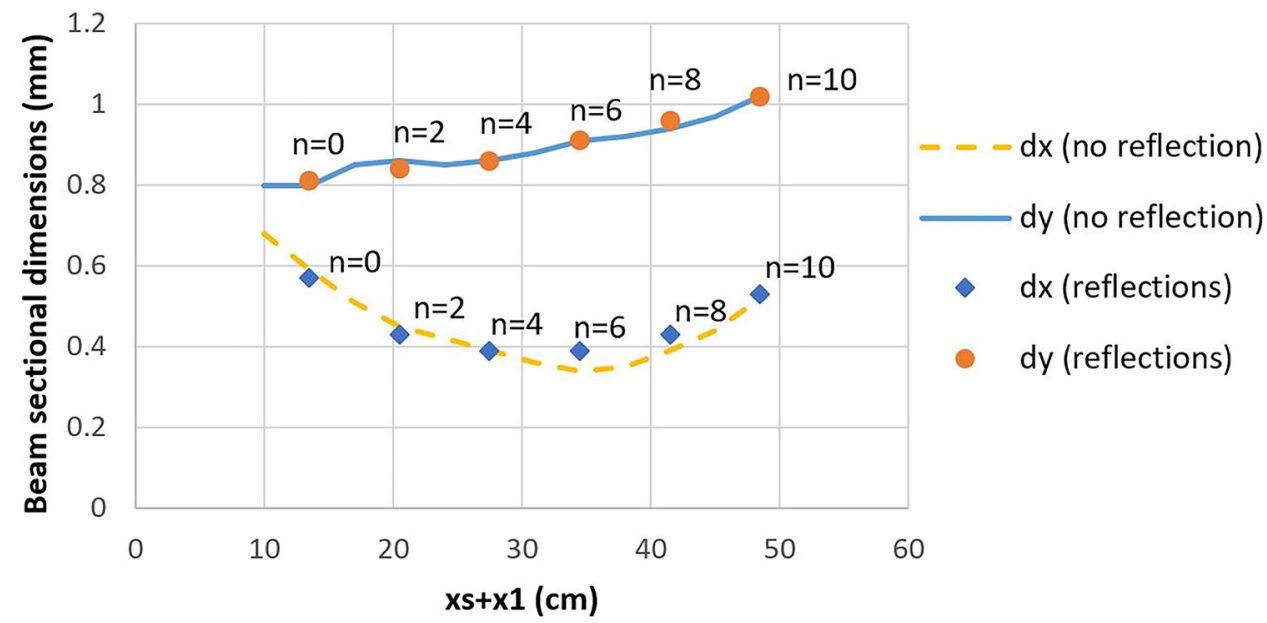

corresponding to $6.8^{\circ}$ with respect to the specimen surface. The optical beam passes at a distance of $60 \mathrm{~mm}$ from the surface of the piece.

Several tests have been carried out starting from $n=$ 0 (i.e., without the mirrors) up to $n=10$, with a distance between the mirrors of $d=35 \mathrm{~mm}$. The trend of the spot as the distance $x_{s}+x_{1}$ from the source varies is shown in Fig. 7, measured with a Gentec EO beam profiler. The figure shows the $d x$ and $d y$ dimensions of the beam obtained both with different reflections between the mirrors and without reflections, as a function of the distance of the laser source from the receiver. The trends are practically superimposed and therefore, even with multiple reflections, the presence of mirrors and diffraction phenomena do not significantly influence the spot diameter.

To maximize the response of the photodetector, the laser power has been varied in each test to compensate for the loss of power caused by reflections on the mirrors and the variation in the spot diameter. Figure 8 shows the trend of photodetector response as a function of the laser power, for diverse numbers of reflections. Optical path lengths are also reported for each tested configuration. By augmenting the number of reflections, an increase in the laser power is required to obtain the maximum response of the system, since a part of the power is absorbed and refracted at each reflection. As a specific value of power is attained, a saturation of the photodiode occurs and response decreases.

As the number of reflections increases, the path within the $x_{s}$-long altered pressure zone widens, also augmenting the system response. Figure 9 depicts such an increase as $x_{s}$ augments, compared with the response obtained by the system in the absence of mirrors. In the case of reflections on mirrors, laser power has been chosen to optimize the response. It can be noted that, with the same $x_{s}$, the response of the system with or without mirrors is practically the same, but with the mirrors, the measurement area remains confined to a small region with a length equal to $35 \mathrm{~mm}$. Data have been obtained employing 64 averages on the signal; the background noise is particularly high for the used instrumentation and is around $70 \mathrm{mV}$.
Fig. 8 Trend of the peak-topeak amplitude of the photodiode signal as a function of laser power and number of reflections on the mirrors

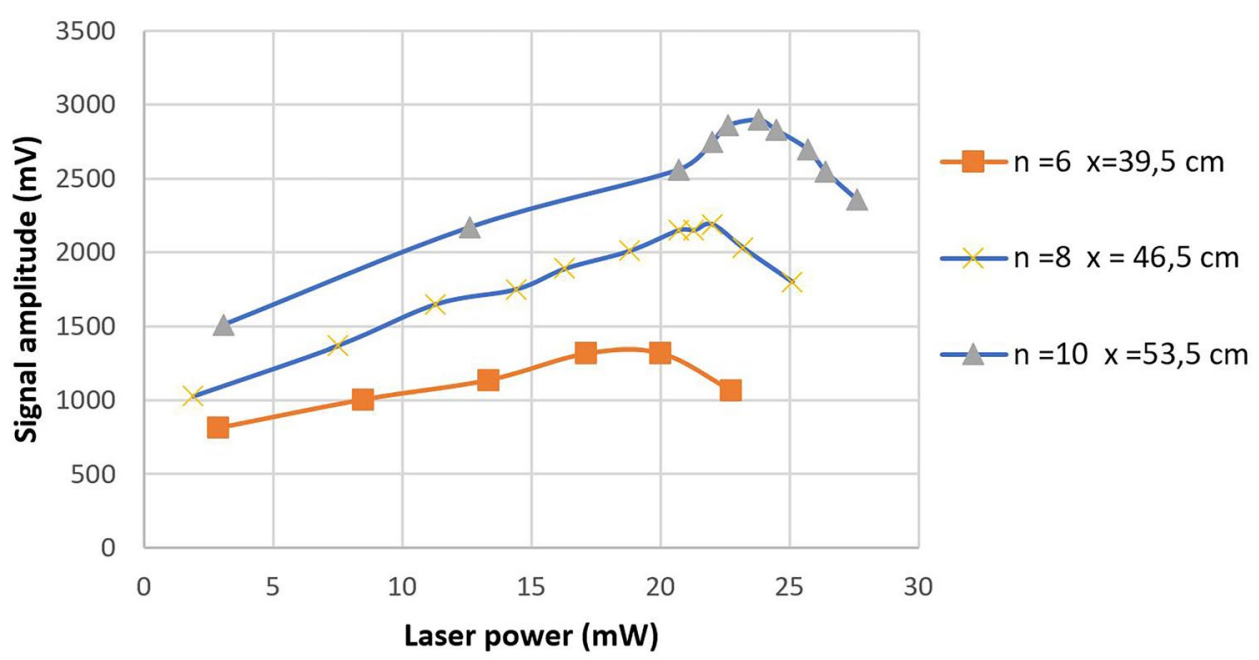


Fig. 9 Response of the system as a function of length $x_{s}$, with and without reflections of the probe laser beam on the mirrors

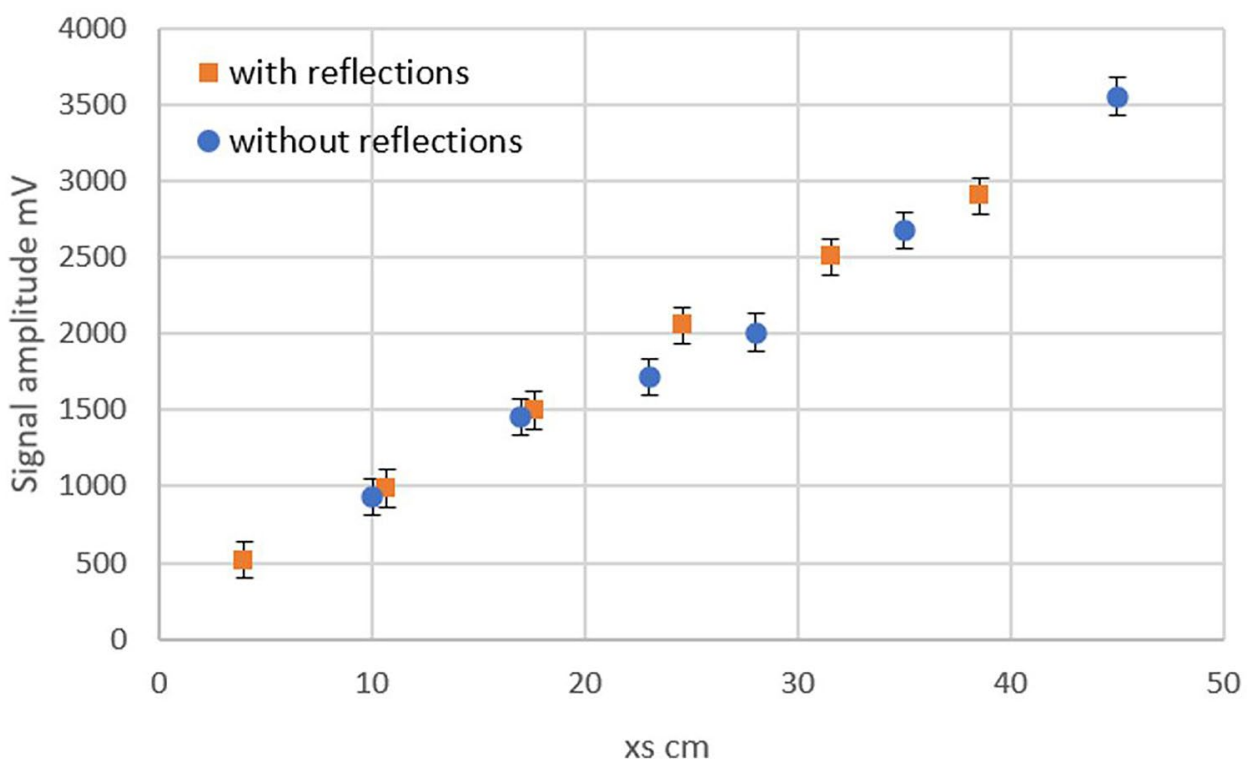

\section{Applications and Discussion}

The preliminary investigations highlighted in Section 4 have been followed by experiments to illustrate the applicability of the developed device. The proposed technique has been employed for the detection of SAWs on a $55 \mathrm{~mm}$ diameter aluminium bar. The choice of a round section bar is more critical than a plate as also the acoustic wavefront in the air is curved: the laser beam, with the use of the proposed experimental apparatus, is found to cross areas with a different pressure gradient both in configuration $\mathrm{A}$ and $\mathrm{B}$ because of the acoustic front curvature; this entails lower sensitivity of the GCLAD technique, as better illustrated in the present Section. Therefore, the choice of a round bar represents a severe test to demonstrate the effectiveness of the proposed method.

The SAW has been generated by a piezoelectric probe at $500 \mathrm{kHz}$, while the equipment is the same employed for tests on a flat bar. Referring to Fig. 3, the application has been performed in both configurations $\mathrm{A}$ and $\mathrm{B}$.

\section{Configuration A}

The layout is illustrated in Fig. 10. As can be seen from the scheme in the side view of Fig. 11, the optical path associated with the various reflections of the laser beam is arranged parallel to the acoustic wavefront in the air. In this way, each section of the beam from one mirror to another is subjected to the same pressure gradient. Since the bar is circular, the wavefront is also circular as shown in the front view of Fig. 11 and is crossed by the optical beam. The overall displacement of the laser beam derives from the integral of all the displacements gradually accumulated along its path; by crossing multiple wavefronts, and therefore areas with opposite sign gradient, the final displacement can also be small [20]. In fact, in the considered case the application of the GCLAD technique without any mirror does not produce appreciable signals, barely visible above the background noise. Conversely, the presence of the mirrors means that the $x_{s}$ path traversed by the laser beam is confined to the area between the mirrors; depending on the distance between the mirrors, the beam may not even cross diverse acoustic wavefronts, which allows obtaining a welldefined, visible signal.

Fig. 12(a) highlights the response trend of the GCLAD system as the length $x_{s}$ increases, resulting from a different number of reflections between the mirrors. The laser power has been adjusted, for each number of reflections, so that the ultrasonic signal is maximized. The curves obtained by testing three solutions with diverse geometry of the area between the mirrors are shown, i.e., $5 \times 20 \mathrm{~mm}^{2}, 15 \times 20$ $\mathrm{mm}^{2}$ and $35 \times 20 \mathrm{~mm}^{2}$. To highlight the influence of the $d$ variable while maintaining approximately the same value of $x_{s}$, Fig. 12(b) depicts two different signals deriving from $d$ values of $15 \mathrm{~mm}$ and $35 \mathrm{~mm}$, and $x_{s}$ values of $28 \mathrm{~mm}$ and $31 \mathrm{~mm}$ respectively.

The size of the active area of $35 \times 20 \mathrm{~mm}^{2}$ allows reaching a greater distance $x_{s}$, but the beam intercepts some wavefronts as shown in Fig. 13; therefore, the response of the system is lower than the other tested solutions. By reducing the distance between the mirrors, the slope of the curves decreases and higher responses are obtained with the same length $x_{s}$.

The distance $d$ between the mirrors can be chosen so that the laser beam does not cross different wavefronts, to obtain a greater response. Obviously, the shorter the distance $d$ 


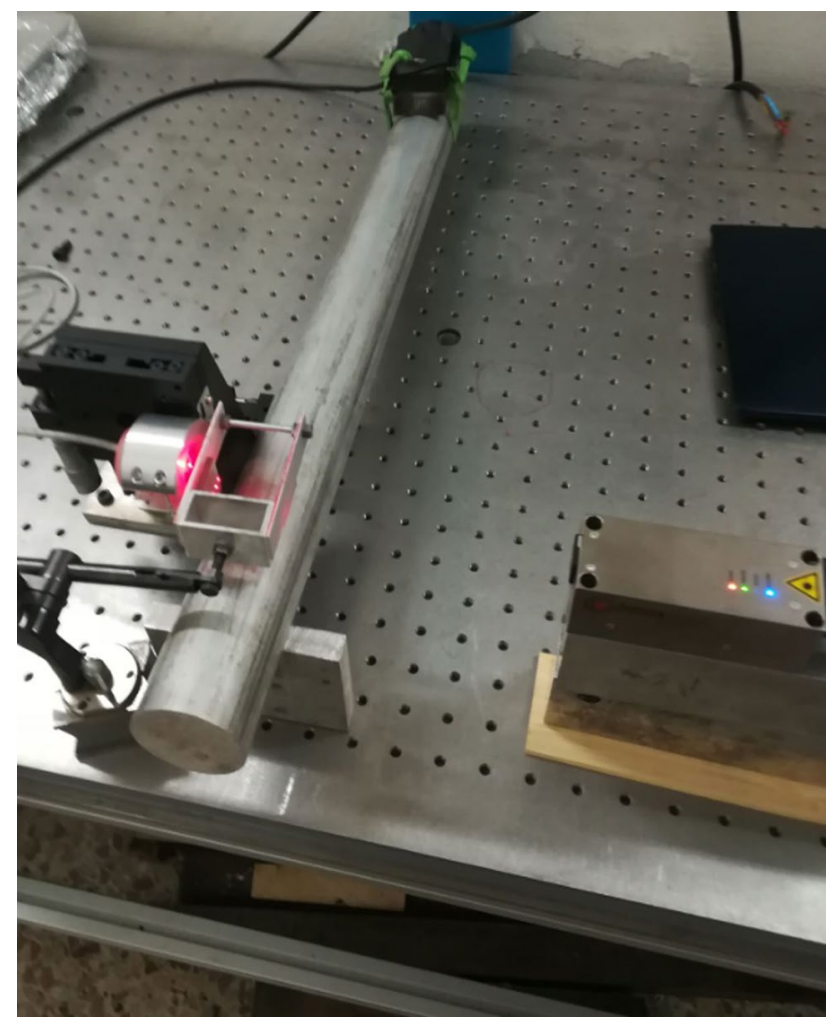

Fig. 10 Configuration A, with the laser beam perpendicular to the bar

between the mirrors, the shorter $x_{s}$ with the same width of the mirrors $h$; the optimal solution must be hence sought through a compromise between the various parameters also depending on the surface of the active area expected to be obtained, i.e., how much the measurement should be accurate. In the tested cases, using $20 \mathrm{~mm}$-wide mirrors and a distance of $5 \mathrm{~mm}$ between the mirrors, a maximum of 18 reflections of the laser beam has been obtained entailing $x_{s}$ $=97 \mathrm{~mm}$. In the case of a distance between the mirrors of 35 $\mathrm{mm}$, a maximum of 14 reflections has been obtained, with a distance $x_{s}=525 \mathrm{~mm}$.

\section{Configuration B}

Configuration B, whose setup is illustrated in Fig. 14, is schematically represented in Fig. 15.

For the tests, a fixed distance $d$ between the mirrors has been employed, equal to $35 \mathrm{~mm}$, while varying the $h$ length. Figure 16 shows the trend of the system response as a function of the $x_{s}$ length, obtained by varying the number of reflections for different lengths $h=10 \mathrm{~mm}, 15 \mathrm{~mm}$ and 20 $\mathrm{mm}$. The measurement chain and the signal amplification values are the same as those used in configuration A. Compared to the results obtained in configuration A, the photodetector response values are significantly higher as the laser beam trajectory between each reflection is parallel to the acoustic wavefront. By reducing the $h$ length, with the same number of reflections or with the same $x_{s}$, an improvement in the signal is obtained. This is caused by the fact that, as can be seen in the front view of Fig. 15, each portion of the optical path between the two mirrors intersects areas with different pressure gradients: if the $h$ length is high, a risk exists that diverse portions of the optical beam will encounter gradients with opposite sign, reducing the total displacement of the laser beam in correspondence of the photodetector. It is hence, in this case, advisable to reduce length $h$ so as to concentrate the passage of all the optical reflections in an area with a pressure gradient as uniform as possible. Essentially, the problem is specular to configuration A.

For small values of $h$, the cylindrical shape of the acoustic wavefronts becomes negligible: with the same $x_{s}$, system responses are obtained which practically equal those obtainable with a single optical beam without reflections on the mirrors. The values achieved with $h=10 \mathrm{~mm}$ are in fact comparable to those obtained on a metal flat bar in which the wavefronts are flat and considering no reflections on the mirrors, as indicated in Fig. 9.

Based on Equations (7)-(8), a maximum of 40 reflections could be obtained without involving beam overlapping on the same mirror from subsequent reflections, considering

\section{Side view}

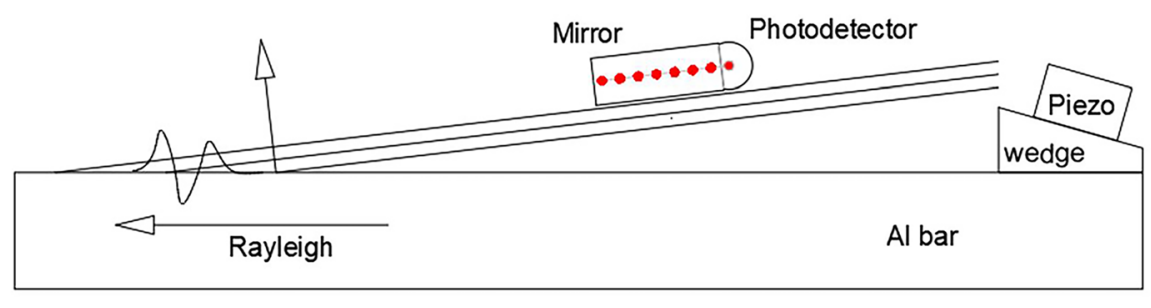

\section{Front view}

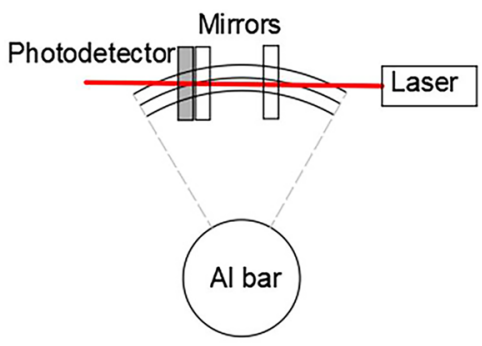

Fig. 11 Scheme of configuration A: the single laser beam reflected between the mirrors intersects the cylindrical acoustic wavefront propagating in the air 
Fig. 12 (a) GCLAD system response as a function of the $x_{s}$ length, obtained by varying the number of reflections of the laser beam between the mirrors for three different geometries of the area between the mirrors - the rectangles at the top right represent the scaled relative dimensions between the three tested solutions, 5 x $20 \mathrm{~mm}^{2}, 15$ x $20 \mathrm{~mm}^{2}$, and $35 \times 20 \mathrm{~mm}^{2}$-; (b) visualization of two different signals acquired by the GCLAD compact probe for exemplarily highlighting the effect associated with $d$ variations, $x_{s}$ being approximately the same (a)
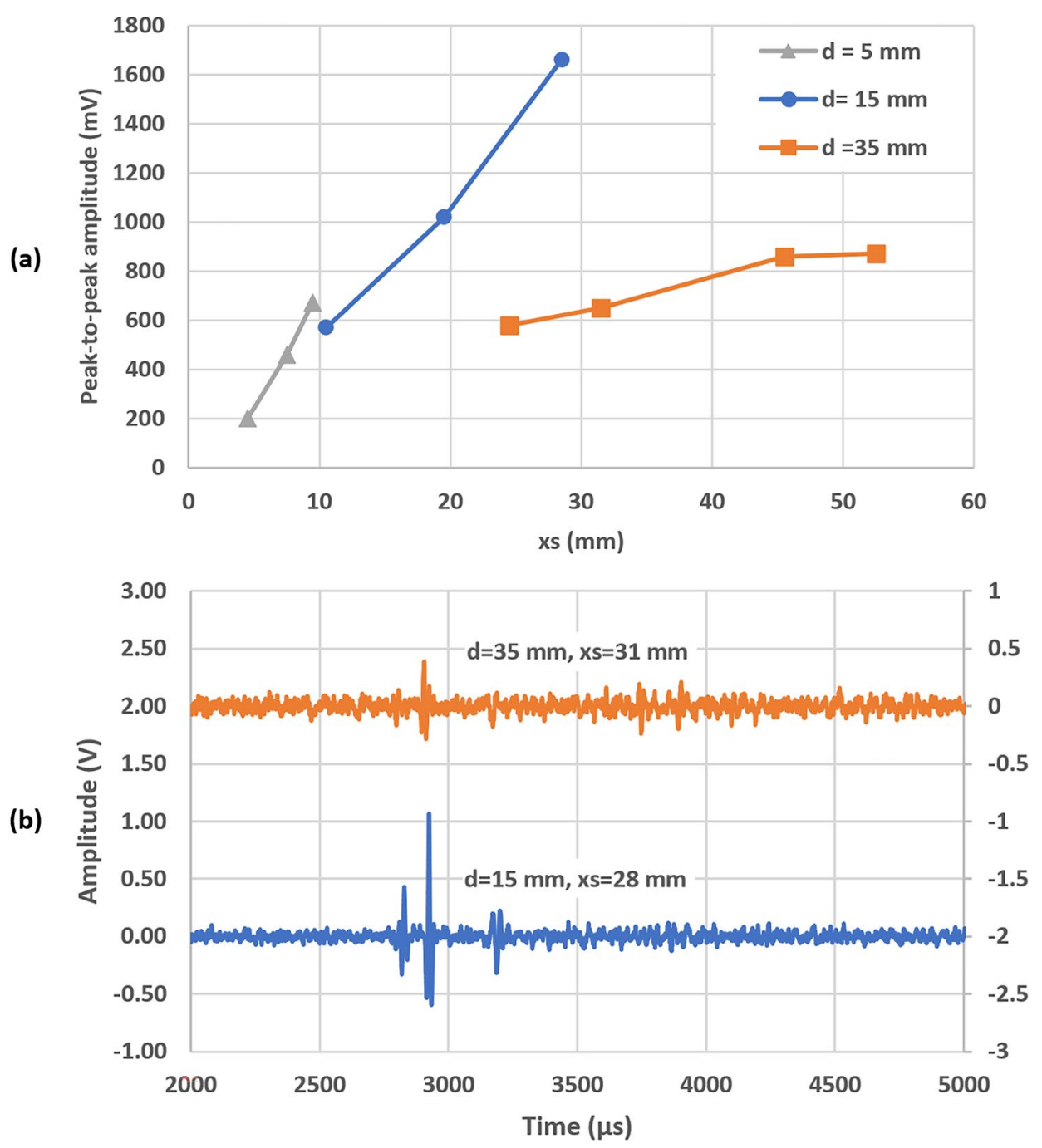

a length of the mirrors equal to $20 \mathrm{~mm}$ and a diameter of the laser beam around $1 \mathrm{~mm}$; practically, this is complex to achieve. The number of reflections obtained without strict alignment and parallelism between the mirrors ranges from 16 to 18 reflections with a distance $d=5 \mathrm{~mm}$, while it

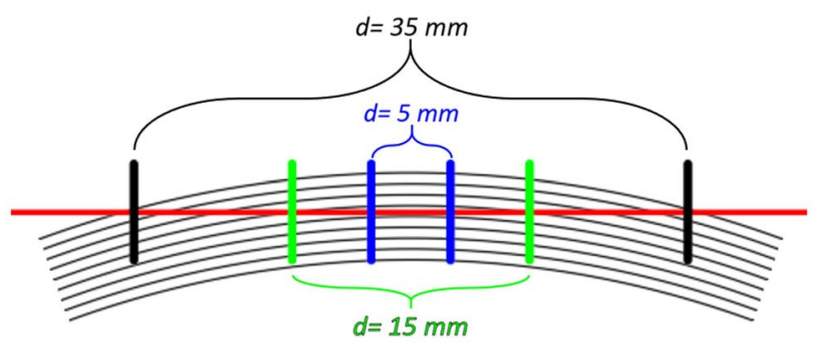

Fig. 13 Representation of the wavefronts in the air crossed by the laser beam, with a wavelength equal to $0.68 \mathrm{~mm}$ (ultrasonic wave frequency of $500 \mathrm{kHz}$ ). The vertical, coloured segments represent the position of the mirrors at a $d$ distance of $5 \mathrm{~mm}, 15 \mathrm{~mm}$ and $35 \mathrm{~mm}$ from each other. With a distance between the mirrors of $35 \mathrm{~mm}$ the laser beam intercepts some wavefronts, differently from the case of the remaining two distances ranges from 12 to 14 reflections for $d=35 \mathrm{~mm}$. This allows obtaining, by optimizing the response of the system, rectangular active areas with a ratio between dimensions more accentuated for configuration B (large distance $d$ and small length $h$ ). For configuration A, a short distance $d$ between the mirrors would be appropriate, increasing the number of reflections through mirrors longer than $20 \mathrm{~mm}$, even if a greater dimension of the active area would result.

To obtain measurements that are as similar as possible to a point detection, therefore ideally with square active areas, configuration $\mathrm{B}$ is the most effective. In this configuration, for tests on a plate, the expected results of the GCLAD system can be obtained from Fig. 9 as a function of the $x_{s}$ employed. For example, for an active area of $15 \times 15 \mathrm{~mm}^{2}$, under the same experimental conditions used to obtain the results of Fig. 9 (test on a flat bar, distance between optical beam and bar $60 \mathrm{~mm}$, acoustic wave generated by the same piezoelectric probe), with 12 reflections an $x_{s}=196 \mathrm{~mm}$ an expected response of approximately $1500 \mathrm{mV}$ is obtained, corresponding to a signal-to-noise ratio equal to 21 . 
Fig. 14 Experimental layout for configuration $\mathrm{B}$, with laser beam parallel to the circular bar

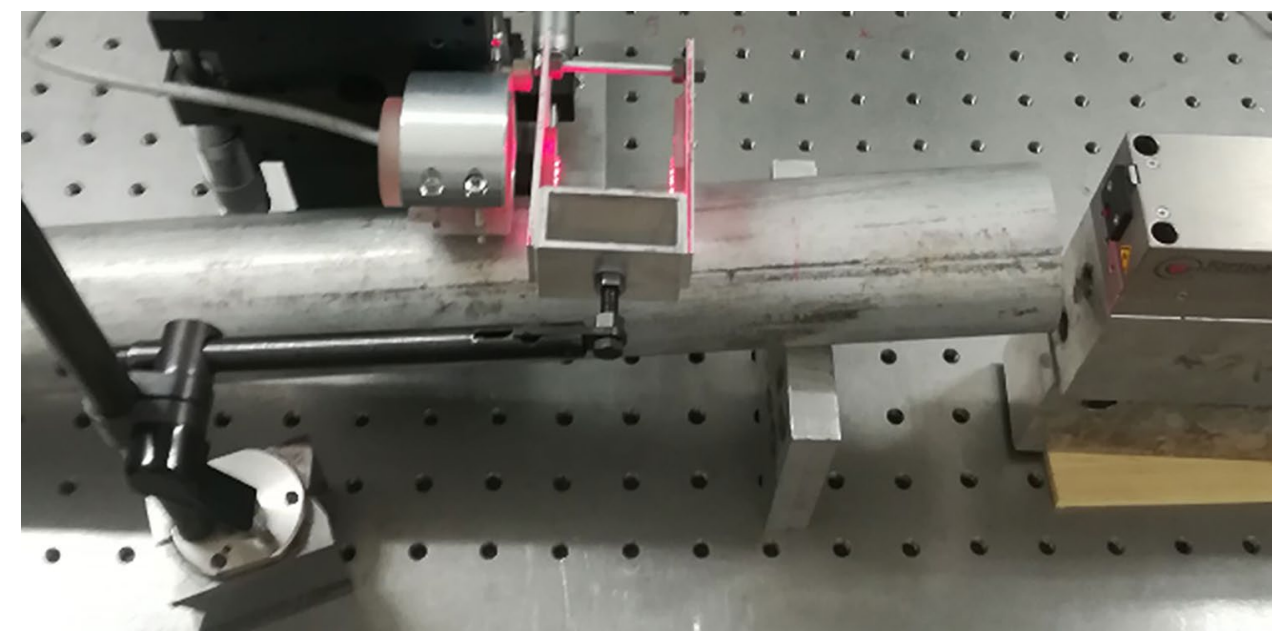

Side view

Front view

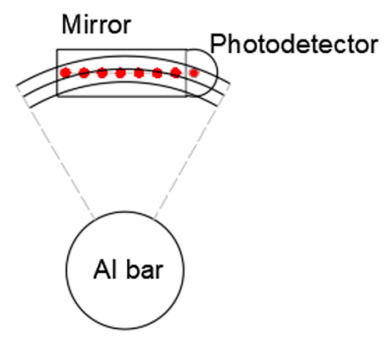

Fig. 15 Scheme of configuration B. The paths between two laser beam reflections run parallel to the acoustic wavefront. Nevertheless, because of the cylindrical shape of the acoustic wavefronts, the single paths intersect areas with different pressure gradients

Fig. 16 Trend of the GCLAD response as a function of the $x_{s}$ length, obtained varying the number of reflections of the laser beam between the mirrors for three diverse $h$ lengths ( 10 $\mathrm{mm}, 15 \mathrm{~mm}$ e $20 \mathrm{~mm}$ )

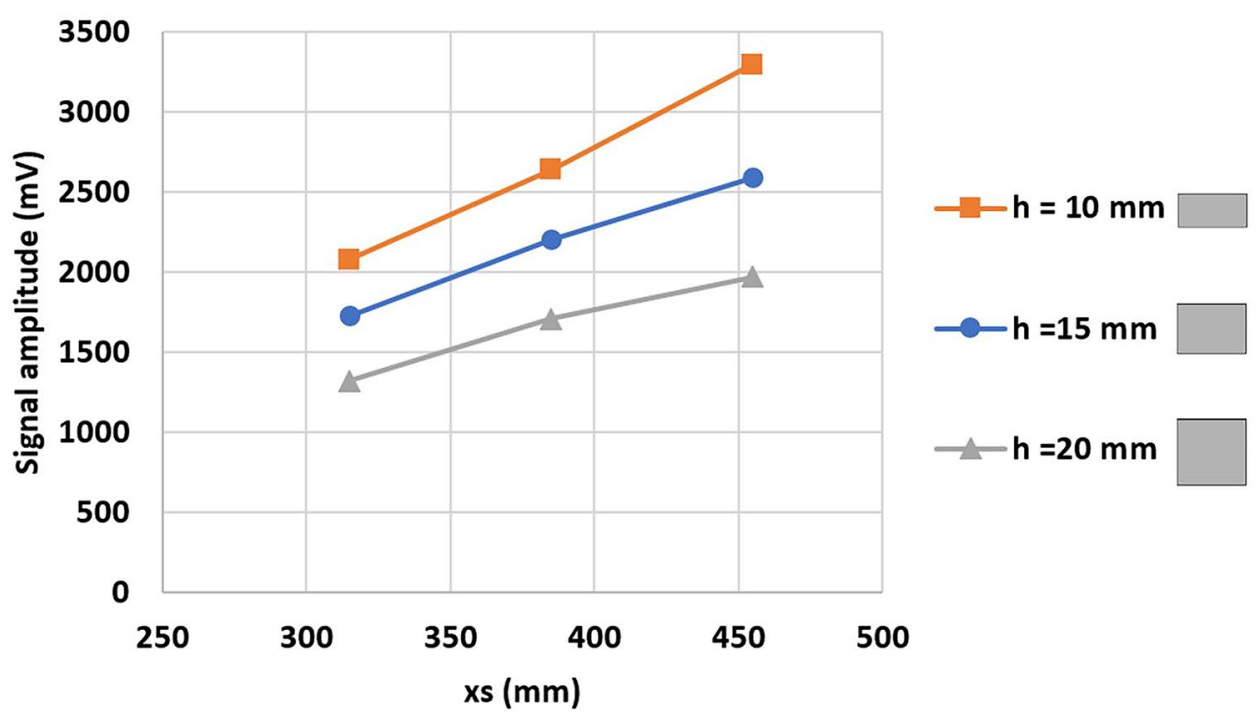




\section{Conclusions}

The GCLAD system is based on the deviation of a laser beam as it travels a fluid with a variable pressure field, like the one generated by diffraction in the air of ultrasonic waves which propagate in a mechanical component. The system permits various experimental configurations for the detection of ultrasonic waves. The study specifically examined two configurations, $\mathrm{A}$ and $\mathrm{B}$, both of which can be used to inspect components that mainly extend in two dimensions with flat or curved surfaces (flat bars or cylinders). Both configurations can be applied with an experimental arrangement comprising two mirrors, to confine the active detection area inside the mirrors. This device, therefore, allows reducing the active detection area and making the non-contact measurement more accurate, like a traditional air-coupled probe or EMAT.

Depending on the distance between the mirrors and their length, rectangular active areas of different geometry and size can be obtained. While for flat surfaces, with flat wavefronts, a wider active area typically corresponds to a greater sensitivity of the method, for curved surfaces with cylindrical acoustic wavefronts the optimal choice is to employ high ratios between the sides of the rectangle defining the active area. Maximum sensitivity can be sought through an appropriate choice of the geometry of the active area and the number of reflections of the laser beam between the mirrors. In the present work, some examples of application to the detection of ultrasonic waves both on a flat bar and on a circular bar have been illustrated, highlighting the influence of the aforementioned parameters on the sensitivity of the method.

The GCLAD, with the application of the described methodology, is suitable for the detection of ultrasonic waves and the non-destructive, non-contact inspection of components. At the state of the art, the technique requires further developments for what regards the design of appropriate processing and filtering circuits, to minimize the noise affecting the measurements.

This work expands the possibilities of using the technique in point-detection investigations on mechanical structures, essential for the accurate localization of defects and NDT application to components characterized by limited accessibility of surfaces ${ }^{1}$.

Acknowledgements The work has been performed as part of the STILUS project (Sviluppo di Tecniche Innovative di controllo nondistruttivo mediante Laser-Ultrasuoni per la Sicurezza degli assili ferroviari), supported by Fondazione CR Firenze.

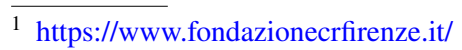

Funding Open access funding provided by Università degli Studi di Firenze within the CRUI-CARE Agreement.

\section{Declarations}

Conflict of Interest The authors declare that they have no conflict of interest.

Open Access This article is licensed under a Creative Commons Attribution 4.0 International License, which permits use, sharing, adaptation, distribution and reproduction in any medium or format, as long as you give appropriate credit to the original author(s) and the source, provide a link to the Creative Commons licence, and indicate if changes were made. The images or other third party material in this article are included in the article's Creative Commons licence, unless indicated otherwise in a credit line to the material. If material is not included in the article's Creative Commons licence and your intended use is not permitted by statutory regulation or exceeds the permitted use, you will need to obtain permission directly from the copyright holder. To view a copy of this licence, visit http://creativecommons.org/licenses/by/4.0/.

\section{References}

1. Caron JN, Yang Y, Mehl JB, Steiner KV (1998) Gas-coupled laser acoustic detection at ultrasonic and audio frequencies. Rev Sci Instrum 69(8):2912-2917. https://doi.org/10.1063/1.1149033

2. Barnes RA, Maswadi S, Glickman R, Shadaram M (2014) Probe beam deflection technique as acoustic emission directionality sensor with photoacoustic emission source. Appl Opt 53(3):511. https://doi.org/10.1364/AO.53.000511

3. Caron JN, Kunapareddy P (2014) Application of gas-coupled laser acoustic detection to gelatins and underwater sensing. In: 40th Annual review of progress in quantitative nondestructive evaluation: Incorporating the 10th International Conference on Barkhausen Noise and Micromagnetic Testing, Baltimore, Maryland, USA, pp 458-463. https://doi.org/10.1063/1.4864855

4. Caron JN, Mehl JB, Steiner KV (1998a) Ultrasonic NDE of Composite Panels with Gas-Coupled Laser Acoustic Detection. In: Thompson DO, Chimenti DE (eds) Review of Progress in Quantitative Nondestructive Evaluation, Springer US, Boston, MA, pp. 635-642. https://doi.org/10.1007/978-1-4615-5339-7_82

5. Požar T, Rus J, Petkovšek R (2017) Optical Detection of Impact Contact Times with a Beam Deflection Probe. Exp Mech 57(8):1225-1238. https://doi.org/10.1007/s11340-017-0289-8

6. Stoklasová P, Grabec T, Zoubková K, Sedlák P, Krátký S, Seiner H (2021) Laser-Ultrasonic Characterization of Strongly Anisotropic Materials by Transient Grating Spectroscopy. Exp Mech. https://doi.org/10.1007/s11340-021-00698-6

7. Caron JN, Mehl JB, Steiner KV (1999) Progress in Gas-Coupled Laser Acoustic Detection for NDE Applications. In: Thompson DO, Chimenti DE (eds) Review of Progress in Quantitative Nondestructive Evaluation, Springer US, Boston, MA, pp 317-324. https://doi.org/10.1007/978-1-4615-4791-4_39

8. Baltazar-Lopez ME (2005) Study of Wave Motion on Tubulars Using Broad-band Laser Ultrasound. Exp Mech 45(5):427432. https://doi.org/10.1177/0014485105056899

9. Dhital D, Lee JR (2012) A Fully Non-Contact Ultrasonic Propagation Imaging System for Closed Surface Crack Evaluation. Exp Mech 52(8):1111-1122. https://doi.org/10.1007/s11340-011-9567-z

10. Johnson JL, van Wijk K, Caron JN, Timmerman M (2016) Gascoupled laser acoustic detection as a non-contact line detector for photoacoustic and ultrasound imaging. J Opt 18(2). https://doi. org/10.1088/2040-8978/18/2/024005 
11. Mihara T, Otsuka Y, Cho H, Yamanaka K (2006) Time-of-Flight Diffraction Measurement Using Laser Ultrasound. Exp Mech 46(5):561-567. https://doi.org/10.1007/s11340-006-8885-z

12. Nuster R, Hofmann-Wellenhof R, Paltauf G (2019) Combined confocal and photoacoustic microscopy based on probe beam deflection technique. In: Oraevsky AA, Wang LV (eds) Photons Plus Ultrasound: Imaging and Sensing 2019, SPIE, San Francisco, United States, p. 171. https://doi.org/10.1117/12.2507502

13. Scruby CB, Drain LE (1990) Laser Ultrasonics: Techniques and Applications. A. Hilger, Bristol, England ; Philadelphia

14. Vangi D, Banelli L, Gulino MS (2021) Interference-based amplification for CW laser-induced photoacoustic signals. Ultrasonics 110. https://doi.org/10.1016/j.ultras.2020.106270

15. Wooh SC, Zhou Q, Wang J (2003) Theoretical and experimental investigation of ultrasonic waves radiated from a wet surface using a line-focused laser beam. Exp Mech 43(4):450-459. https://doi. org/10.1007/BF02411349

16. Vangi D, Bruzzi M, Caron JN, Gulino MS (2021b) Crack detection with gas-coupled laser acoustic detection technique. Measur Sci Tech 32(9):095202. https://doi.org/10.1088/1361-6501/abfced

17. Gulino MS, Bruzzi M, Vangi D (2021) Gas-Coupled Laser Acoustic Detection technique for NDT of mechanical components. Ultrasonics 114. https://doi.org/10.1016/j.ultras.2021.106415

18. Liu X, Yang S, Liu Y, Chi Y, Gu X (2018) Surface Crack Identification on a Cylinder Using the Signal Enhancement of the Scanning Laser Line Source Method. Appl Sci 8(10):1796. https://doi. org/10.3390/app8101796

19. Sohn Y, Krishnaswamy S (2004) Interaction of a scanning lasergenerated ultrasonic line source with a surface-breaking flaw. The Journal of the Acoustical Society of America 115(1):172-181. https://doi.org/10.1121/1.1630997
20. Diaci J (1994) Transfer function of the laser beam deflection probe for detection of cylindrical acoustic waves in a transverse arrangement. Le Journal de Physique IV 04(C7):C7-773-C7-776. https:// doi.org/10.1051/jp4:19947182

21. Cavuto A, Martarelli M, Pandarese G, Revel G, Tomasini E (2015) Experimental Investigation by Laser Ultrasonics for High Speed Train Axle Diagnostics. Ultrasonics 55:48-57. https://doi.org/10. 1016/j.ultras.2014.08.010

22. Caron JN, Thompson DO, Chimenti DE (2008) Displacement and deflection of an optical beam by airborne ultrasound. In: AIP Conference Proceedings, AIP, Golden (Colorado), vol 975, pp 247-254. https://doi.org/10.1063/1.2902666

23. Johnson JL, van Wijk K, Caron JN, Timmerman M (2016b) Photoacoustic and ultrasound imaging with a gas-coupled laser acoustic line detector. In: Oraevsky AA, Wang LV (eds) SPIE BiOS, San Francisco, California, United States, p. 970814. https://doi. org/10.1117/12.2211800

24. Towne DH (2014) Wave Phenomena. Dover Publications, New York

25. Yariv A et al (1997) Optical Electronics in Modern Communications, vol 1. Oxford University Press, USA

26. Gulino MS, Bruzzi M, Vangi D (2020) Application of the gascoupled laser acoustic detection technique to non-destructive testing of mechanical components. In: XXVIII AIVELA Conference, Online, $\mathrm{p}$ (In press)

27. Chung CH, Lee YC (2013) An Improved Weighting Method for Inversely Determining Elastic Constants of Coating Layers from Dispersion Curves of Surface Acoustic Waves. Exp Mech 53(8):1395-1403. https://doi.org/10.1007/s11340-013-9739-0

Publisher's Note Springer Nature remains neutral with regard to jurisdictional claims in published maps and institutional affiliations. 